\title{
Filigrane
}

Écoutes psychanalytiques

\section{Narcissisme blessé et identité en souffrance à l'adolescence}

\section{Pascal Roman}

Volume 28, numéro 1, 2019

URI : https://id.erudit.org/iderudit/1064598ar

DOI : https://doi.org/10.7202/1064598ar

Aller au sommaire du numéro

Éditeur(s)

Santé mentale et société

\section{ISSN}

1192-1412 (imprimé)

1911-4656 (numérique)

Découvrir la revue

Citer cet article

Roman, P. (2019). Narcissisme blessé et identité en souffrance à l'adolescence. Filigrane, 28(1), 75-87. https://doi.org/10.7202/1064598ar

\section{Résumé de l'article}

Le processus pubertaire ouvre des remaniements importants dans la construction identitaire, sur le fond des remaniements narcissico-objectaux à l'adolescence. Les violences sexuelles à l'adolescence peuvent être considérées comme un marqueur d'une faillite des réaménagements pubertaires, dans le contexte de l'actualisation traumatique d'un narcissisme blessé. Après l'exposé de perspectives théoriques sur l'identité dans son rapport au narcissisme, la présentation du traitement psychanalytique d'un adolescent permet d'aborder les enjeux d'une identité en souffrance. 


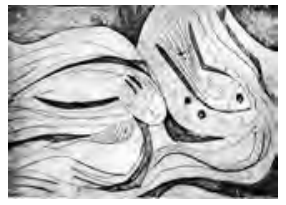

\title{
Narcissisme blessé et identité en souffrance à l'adolescence
}

\author{
Pascal Roman
}

\begin{abstract}
Résumé: Le processus pubertaire ouvre des remaniements importants dans la construction identitaire, sur le fond des remaniements narcissico-objectaux à l'adolescence. Les violences sexuelles à l'adolescence peuvent être considérées comme un marqueur d'une faillite des réaménagements pubertaires, dans le contexte de l'actualisation traumatique d'un narcissisme blessé. Après l'exposé de perspectives théoriques sur l'identité dans son rapport au narcissisme, la présentation du traitement psychanalytique d'un adolescent permet d'aborder les enjeux d'une identité en souffrance.
\end{abstract}

Mots clés: identité; adolescence; narcissisme; violences sexuelles; traumatisme.

\begin{abstract}
The process of puberty opens up significant changes in the construction of identity, on the background of the changes of the narcissistic-object during adolescence. Sexual violence in adolescence can be considered as a marker of a failure of puberty rearrangements, in the context of the traumatic re-actualization of an injured narcissism. Following the presentation of theoretical perspectives on identity in its relation to narcissism, the presentation of the psychoanalytic treatment of teenagers allows for a clarification of the stakes at play in a suffering identity.

Key words: identity; adolescence; narcissism; sexual violence; trauma.
\end{abstract}

$\mathrm{N}$ otre référence au "narcissisme blessé» est empruntée aux travaux de Ciavaldini (2006) qui met l'accent sur les graves atteintes au narcissisme qui caractérisent la vie psychique des auteurs de violences sexuelles, particulièrement lorsque les victimes sont prépubères. Ciavaldini propose de référer la notion de narcissisme blessé aux auteurs d'actes pédophiles au regard de la précarité dans la construction des auto-érotismes, dont on connaît, depuis Freud (1905), la contribution essentielle à l'élaboration du narcissisme. La figure clinique du narcissisme blessé peut être considérée à partir de l'hypothèse d'un défaut de la qualité des premières relations: «En effet, ces sujets ont souffert d'une discontinuité excessive d'avec la mère primaire, ce que D. Bouchet-Kervella nomme un déficit en "pédophilie primaire" nécessaire à la constitution de l'assise narcissique de l'enfant»(Civaldini, 2006, p. 182). 
Or le temps de l'adolescence place sur le devant de la scène les variations dans l'équilibre narcissico-objectal aux prises avec le jeu des remaniements identitaires et identificatoires (Jeammet, 1994). Gutton (1991) décrit en particulier les aléas du traitement de la figure de l'auto-séduction à l'adolescence, qui apparaît sur la voie de la résolution du traumatisme pubertaire comme le témoin de la mise en crise des contours de l'identité. C'est dans ce contexte que la préoccupation pour la qualité des assises narcissiques de l'adolescent et leur remise en jeu, témoin de la refondation identitaire, mobilise le psychothérapeute dans son compagnonnage soignant.

Narcissisme et identité ont partie liée. À la suite des travaux de Lacan portant sur la description du stade du miroir (1949), puis de Winnicott (1956) sur la notion de self, la fonction structurante du narcissisme se trouve affirmée, au service du développement de l'unité du moi, dont on peut relever la contribution centrale à l'identité. Dans ce contexte, le narcissisme peut être compris comme le fond processuel sur lequel se développe l'identité, dans ses qualités fondatrices d'unicité, de continuité et de permanence (Laplanche et Pontalis, 1967).

Plus proches de nous, les apports de Cahn (2004) permettent de préciser, tout spécialement dans le temps de l'adolescence, les conditions dans lesquelles se trouvent remises au travail l'identité et les identifications, dont il est indispensable de considérer l'étroite intrication dans leur déploiement. Avec la notion de subjectivation, Cahn nous offre tout à la fois une occasion de penser la part réflexive constitutive de la construction identitaire et la contribution de l'objet au développement des processus qui sous-tendent les réaménagements adolescents. C'est en effet dans la dialectique ouverte entre subjectivation et subjectalisation que peut être considérée la dynamique identitaire à l'œuvre, et les éventuels ratés de celle-ci.

\section{Malvin, une identité en souffrance}

Au fil du travail psychothérapeutique engagé avec Malvin, il m’est assez clairement venu à l'esprit que celui-ci, âgé de 15 ans au moment où je le rencontre, représente la figure d'un adolescent au narcissisme blessé. Malvin est né d'une mère originaire d'un autre continent et d'un père européen. La rencontre clinique avec Malvin s'inaugure par une demande de la mère de l'adolescent qui sollicite un rendez-vous dans une consultation spécialisée, car son fils vient de contraindre de jeunes garçons prépubères, voisins habitant le même immeuble, à des actes d'ordre sexuel (il a réalisé sur eux des fellations, en marge d'une fête qui réunissait les résidents du quartier). 
L'accompagnement psychothérapeutique de Malvin (à un rythme hebdomadaire pendant un peu plus d'une année, à quinzaine au-delà) se transformera dans les mois qui suivent, après jugement, en une obligation judiciaire de soin.

Malvin a beaucoup de difficultés à comprendre la dimension de transgression de ses actes, comme s'ils étaient hors de tout éprouvé sensible, mais également hors du temps, hors de l'espace et donc hors de toute histoire. Malvin se présente comme un adolescent très dépendant de sa mère (son père est décédé lors de sa toute petite enfance), en léger surpoids, et toujours fatigué, présentant les traits de ce que l'on pourrait identifier comme un symptôme d'hypersomnie à l'adolescence. Par ailleurs, il est notable que la tonalité de l'engagement homosexuel des agirs violents sexuels qui lui sont reprochés n'affleure d'aucune manière dans les premiers échanges avec Malvin.

J'apprends assez rapidement que Malvin a été confié à sa grand-mère maternelle dans le pays d'origine de sa mère alors qu'il était âgé de quelques mois. Sa mère, émigrée en Europe, considérait qu'elle ne pouvait plus prendre soin de lui de manière satisfaisante, alors que son père, malade, nécessitait des soins permanents de sa part. Ce dernier décède d'un accident de la circulation alors qu'il rend visite à Malvin, quelques mois après que ce dernier ait quitté l'Europe.

Malvin grandira dans la famille maternelle (grand-mère, tantes et oncles, cousines et cousins) pendant plusieurs années. Il reviendra ensuite vivre auprès de sa mère restée en Europe: il y découvre le nouveau mari de la mère et un bébé né entre-temps, et passera la fin de la période de latence et le début de l'adolescence au nouveau domicile familial de sa mère. Pendant cette période et plusieurs années durant, Malvin se dit victime de harcèlement à l'école: il fait l'objet de moqueries liées à son aspect physique et se trouve en position d'exclusion de la part de ses camarades. Il est alors à nouveau confié à la famille maternelle pendant 18 mois hors d'Europe, "pour le protéger» précise sa mère. Lorsqu'il revient en Europe, à sa demande expresse et avec un engagement de sa part pour que "tout se passe bien», Malvin fait état d'une souffrance importante vécue chez sa grand-mère maternelle: il a souffert d'une absence de préoccupation à son égard, de différentes contraintes auxquelles il est soumis, mais aussi de la proximité avec une grand-mère qu'il vit comme abusive dans ses exigences éducatives. C'est quelques semaines après son retour au domicile familial qu'il commet des actes d'ordre sexuel sur de jeunes garçons, faits qui feront l'objet d'une 
dénonciation de la part des parents de ces derniers, puis d'une poursuite et d'une condamnation judiciaire.

\section{L'idéalisation au service du gel du pubertaire}

Le travail de psychothérapie avec Malvin est laborieux: l'adolescent ne se souvient de rien, ni de son enfance sur le continent extra-européen où il a vécu, ni des actes qui lui sont reprochés et qu'il ne conteste pas, ni des motivations qui ont pu être les siennes. Son discours est lisse: « tout va bien» pour lui, la famille maternelle recomposée est présentée comme sans faille. La figure maternelle est idéalisée, dans un registre tout à la fois héroïque et sacrificiel, alors que la figure paternelle se trouve rabattue sur la figure du beau-père: son beau-père est son père, sans jeu significatif entre ces deux références.

Par ailleurs, tout se passe comme si Malvin n'était pas entré en adolescence et que, d'une certaine manière, la question ne se posait pas. On peut bien sûr identifier quelques conflits avec sa mère ou son beau-père autour de l'achat ou l'usage des consoles de jeu et du téléphone portable. Si ces conflits sont à la fois vécus de manière intense sur le plan affectif et émotionnel (ils sont l'occasion de mettre en question l'amour que son beau-père lui porte), ils sont dans le même temps considérés par Malvin comme dérisoires.

La puberté semble avoir glissé sur Malvin sans l'atteindre: son léger surpoids contribue à masquer les transformations corporelles, sa voix même parait ne pas avoir mué. Malvin se présente par ailleurs dans une compliance relationnelle parfois agaçante du fait des codes très formels qu'il emprunte pour s'adresser, et qui semblent mobiliser alternativement et conjointement des mouvements de dépendance et/ou de séduction. On peut avoir le sentiment que les remaniements pulsionnels liés à la puberté n'ont pas pu prendre corps chez Malvin et que ses objets d'investissement sexuel, dans un registre génitalisé, sont inexistants. Le gel du processus du pubertaire (Gutton, 1991) ${ }^{1}$ apparaît sous-tendu par l'insécurité essentielle dans laquelle a grandi Malvin et qui a teinté le développement de son self, ainsi que par l'impasse dans laquelle le précipitent les fantasmes d'auto-séduction dont on peut identifier une mise en acte au travers des agirs violents sexuels sur de jeunes garçons: pour qui compte-t-il et sur qui peut-il compter? Comment peut-il construire sa place au sein d'une famille qui alternativement le rejette et l'accueille, éventuellement sous condition (on se rappelle que la décision de son retour en Europe à 15 ans était conditionnée par son engagement à ce que «tout se passe bien»)? Comment s'engager dans un 
devenir adulte qui exige le traitement d'un deuil de l'infans auquel Malvin ne semble pas avoir accès pour l'heure?

En effet, tout se passe comme si le gel du pubertaire maintenait vivant pour Malvin un registre de sexualité infantile, au service de la satisfaction immédiate, sur le fond traumatique dont témoigne son histoire. Malvin peut en effet nommer des satisfactions infantiles, partielles, avec les jeux vidéo par exemple, qui représentent un espace dans lequel il paraît en mesure d'exister et de se valoriser. Au-delà, les investissements adolescents de Malvin ne semblent pas pouvoir prendre appui sur l'investissement d'un sexuel infantile suffisamment consistant et satisfaisant. Par ailleurs, le vécu de "désaide» (Freud, 1926) et de défaut d'une protection de la part des adultes ouvre une forme de béance au sein de tout processus de mise en sens de l'histoire de Malvin et des éprouvés qui y sont attachés: en effet, on peut identifier des failles dans le travail de symbolisation, qui s'expriment au travers des échappées de sens qui émaillent son discours, lorsqu'un mot convoqué pour un autre s'impose dans le discours, ou lorsque l'échange concerne des aspects financiers ou comptables, qui impliquent la mise en œuvre d'opérations logiques dont le sens semble se dérober à lui.

Le lien à la mère est marqué par une grande proximité, dans un contexte où Malvin se reproche les "problèmes» qu'il a occasionnés au sein de sa famille. Malvin pourra au fil de la psychothérapie évoquer que sa mère et son beau-père se disputent à cause de lui, qu'ils sont en conflit avec leurs voisins du fait des agirs sexuels transgressifs sur leurs enfants. Par ailleurs, Malvin soutient que le jeune enfant de sa mère et de son beau-père n'est pas au courant de ces agirs, tout en reconnaissant que celui-ci est affecté par les exigences des parents de ne plus inviter qui que ce soit à la maison; Malvin, quant à lui n'a plus le droit de sortir du domicile familial, hormis pour suivre sa scolarité. Dans ce contexte, émerge la figure d'un secret dont je comprendrai par la suite que la fonction pourrait bien être d'en masquer un autre sur le mode: «Un secret peut en cacher un autre ${ }^{2}$.» On peut entendre que l'énoncé du secret (qui, pour une part, porte atteinte à la consistance identitaire de Malvin puisqu'il contribue à annuler les agirs dont il est l'acteur) fait signe d'autres modalités de secret dans la transmission qui échappent pour une large part au travail psychothérapeutique.

Quel serait alors ce secret? Celui de la défaillance maternelle à laquelle Malvin doit non seulement survivre dans ses investissements infantiles mais qu'il doit également pallier secondairement, dans l'actuel, sur le mode de la répétition? Ou celui de la charge incestueuse ouverte par l'assignation à une 
forme de complément narcissique maternel dans le contexte d'une réparation traumatique d'un vécu de lâchage précoce?

Pendant plusieurs mois, Malvin met en question le sens de l'obligation de soin, tout en livrant progressivement des éléments du présent de son histoire: il doit prendre soin de sa mère dans des épisodes où elle s'absente, sans pouvoir compter sur le soutien de son beau-père. Malvin se trouve dans ce contexte contraint d'investir une position de substitut maternel lorsque sa mère n'est pas en mesure d'assurer les soins primaires à son jeune enfant: repas, toilette, sommeil... C'est à partir de l'énoncé, longtemps masqué dans les séances, de cet éprouvé d'être débordé par les tâches nécessitées du fait de l'incapacité maternelle vis-à-vis de ce dernier que Malvin sera en mesure d'aborder prudemment son propre vécu d'abandon à l'égard de sa mère, dans le contexte de la discontinuité des soins maternels: vécu d'abandon dans l'actuel, mais aussi dans sa petite enfance et son adolescence. Il a dû prendre soin de lui-même, sans recours, sans secours.

Le travail de psychothérapie visera alors à accompagner les mouvements de désidéalisation de la figure maternelle, pour tenter de retrouver au sein de la vie psychique, les traces de l'espoir dont Winnicott indique qu'il se trouve contenu dans la tendance antisociale: "Dans la tendance antisociale, l'espoir est sous-entendu» (Winnicott, 1956, p. 149). Il s'agit en effet, dans le cadre de la psychothérapie, de permettre à Malvin de faire l'expérience d'une figure de soutien suffisamment inconditionnelle, sorte de prothèse pour lui permettre de re-construire une sécurité de base, sans pour autant méconnaître la dimension délétère de ses transgressions à l'égard des victimes et de lui-même. Au plan transférentiel, l'écho de la compliance et des positions soumises de Malvin résonne dans un vécu d'impuissance ou d'agacement, face au sentiment de l'absence d'épaisseur de ses inscriptions historiques et de l'écart entre sa soumission et le retrait de son investissement dans le lien psychothérapeutique. Un des écueils identifiés dans la maturation des remaniements narcissiques et identitaires de Malvin concerne, on l'a dit, le gel du processus pubertaire. Envisagé d'un autre point de vue, sans doute pourrait-on parler d'un gel du travail de deuil lié au nécessaire détachement des objets de l'enfance et de la qualité des liens qui y sont attachés. Lorsque les objets de la période infantile sont trop incertains, ou instables, ou peu sécurisants comme cela est le cas pour Malvin, le processus de détachement, préalable à de possibles ré-investissements, se trouve empêché, entravé. J'ai évoqué précédemment que chez Malvin la puberté semblait ne pas avoir fait trace, ainsi qu'en témoigne sa manière de se présenter dans la relation à 
l'autre sur un mode infantile, tant dans son habillement que dans le rapport de soumission à l'autorité que représente l'adulte. L'hypothèse d'une enclave mélancolique peut à cet endroit être évoquée, en lien à un objet encrypté, objet maternel introuvable et à jamais perdu auquel Malvin s'identifie massivement. Il est intéressant de relever de quelle manière cet objet apparait comme étant pris dans le clivage: tout à la fois idéalisé, intouchable, porté par la figure d'une mère parfaite et héroïque, et attaqué, dénigré en lien à l'incapacité de cette dernière à prendre soin (de lui ou du jeune enfant du couple). Lors d'une séance avec ses parents et l'éducateur du Tribunal des mineurs, qui se déroule après environ 18 mois de psychothérapie, Malvin peut mentionner son inquiétude face aux épisodes de défaillance de la capacité de soutien de sa mère et de son beau-père, inquiétude dont on peut envisager qu'elle vient faire écho aux vécus infantiles d'abandon. Cette capacité de soutenir une parole signe une forme d'autonomisation à l'égard de la dépendance aux objets parentaux, et porte la marque d'un cheminement sur la voie de la subjectivation. Dans le même temps, il est important de noter que Malvin continue à dénier la pertinence du soin sous mandat judiciaire, soin qu'il parvient cependant progressivement à investir de manière adéquate. Ces différents éléments sont le témoin de la précarité d'un travail de deuil des objets de l'enfance jusque-là impossible, dans la mesure de la confusion qui préside à l'investissement de l'objet maternel dans une figure idéalisée-destructrice: le travail du clivage peut, dans ce contexte, faire signe d'un jeu possible entre les deux parts aliénées du moi, et ouvrir la voie à l'ambivalence.

\section{Le deuil de l'infans et la subjectivation}

Dans ce contexte, les perspectives ouvertes par le traitement psychothérapeutique concernent trois aspects conjoints:

- l'accompagnement de Malvin dans le processus, irrémédiable, du deuil de l'infans dont l'engagement paraît en suspens: il s'agit de soutenir l'adolescent dans son engagement dans le processus d'adolescence, pour une part évité, dénié, au profit de la survivance de liens de dépendance. L'agir violent sexuel fait à ce titre événement, au sens où le propose Marro (2019), évènement susceptible de marquer, en lieu et place de la puberté, l'amorce du processus adolescent $^{3}$;

- l'écoute de l'infans dans l'adolescent, avec l'accueil des parts les plus archaïques de sa vie psychique: cela nécessite d'entendre la part 
d'infans au cour de la vie psychique de Malvin, part souffrante et carencée, et d'accueillir de manière inconditionnelle, sans rétorsion, la part de souffrance liée au narcissisme blessé, y compris dans les manifestations régressives qui émergent en séance (Malvin glisse du fauteuil, se plaint de la fatigue et fait mine de s'endormir...);

- la mobilisation de la figure du répondant (Kaës, 2012), figure de celui qui est concerné par l'adolescent et qui accepte de se tenir face à lui: ici le répondant vient signifier la fonction structurante de l'environnement, en ce qu'il témoigne tout à la fois d'une compréhension et d'une limite, en contrepoint de la forme d'indifférence à laquelle peut renvoyer le positionnement parental, envahi par ses propres angoisses et souffrances, et marqué par la discontinuité de sa préoccupation. On peut considérer que le soin sous contrainte contribue à cette figure du répondant (Roman, 2018) et autorise Malvin à jouer avec la passivité à laquelle il est contraint, dans une forme de réplication du processus pubertaire.

Comment comprendre alors les enjeux du travail de subjectivation (Cahn, 1998) dans sa contribution au soutien des remaniements de l'identité de Malvin? Malvin peut parfois énoncer la confusion dans laquelle il se trouve au regard des premières figures de soin: «qui est ma mère?» On peut entendre: «sur quelle figure porte le choix d'objet par étayage, dans le contexte des vécus de discontinuité de l'objet-support?» À cet égard, on peut faire l'hypothèse d'un surinvestissement du choix d'objet narcissique, à même de protéger le propre narcissisme blessé de l'adolescent. Ce surinvestissement conduit Malvin à se maintenir dans un lien infantile à l'objet, contribuant à tenir à distance les nécessaires remaniements identitaires initiés par la puberté. Par ailleurs, Malvin paraît se situer en retrait du conflit d'ambivalence lié au jeu identificatoire, comme en témoignent l'idéalisation des figures parentales et la confusion des figures paternelles et beau-paternelles sur lesquelles Malvin ne s'arrête à aucun moment, y compris lorsque je relève les effets de confusion dans ma compréhension de ses propos. Dans ce sens, le traitement psychothérapique soutient un possible mouvement de conflictualisation, qui s'autorise à émerger progressivement, et dont on peut repérer la trace au travers des récits de Malvin, qui témoignent de son investissement progressif de la relation à ses pairs, soutien pour le développement d'identifications latérales.

Une autre voie d'expression des fragilités narcissiques et identitaires de Malvin peut être considérée à partir de son engagement dans les agirs 
violents sexuels qui ouvrent à la rencontre clinique: en effet, on peut faire l'hypothèse d'une quête de l'adolescent d'une confirmation narcissique et identitaire dans le corps-à-corps imposé aux victimes. Les violences sexuelles commises à l'encontre d'enfants prépubères constitueraient ainsi une figure actualisée de l'auto-séduction (Gutton, 1991), au sens de la mise en scène dans la réalité externe d'un conflit pulsionnel qui ne trouverait pas à se résoudre sur la scène interne: les victimes se présenteraient alors comme des doubles projetés de Malvin, sur le mode de la duplication projective (Roman et Ravit, 2006), doubles dont la caractéristique consiste dans une faible différenciation entre sujet et objet. On se rappelle que Malvin reconnaît les faits pour lesquels il est mis en cause, puis condamné, mais qu'il ne peut rien en dire, ni rien dire des garçons qu'il a contraints à des actes sexuels, de leurs éprouvés et des siens.

Au-delà, on pourrait penser que le traumatisme pubertaire est venu réactiver les traumatismes précoces (séparation d'avec la mère, insatisfaction des relations de maternage, décès brutal du père, harcèlements), laissant Malvin désemparé face aux bouleversements corporels et psychiques de la puberté, débordé par les mouvements d'auto-séduction qui l'agitent. On peut entendre que les violences sexuelles contre les garçons témoignent davantage d'une tentative de réparation d'une blessure narcissique liée à la transformation du corps (sur le mode d'une identification primaire, homomorphe) que de l'expression d'un choix d'objet homosexuel agi sur un mode transgressif.

\section{Confortation identitaire et déploiement d'une fantasmatique génitale}

On a pu repérer l'apparent désert de l'investissement de la sexualité génitale chez Malvin, alors que les violences sexuelles infligées aux victimes, d'allure génitale, s'inscrivent dans une modalité prégénitale et, à ce titre, dans une modalité partielle de la satisfaction sexuelle, qui échappe à l'inscription au sein d'un partage affectif: modalité orale, sur le mode de la contrainte et sur le fond d'un déni du désir de l'autre. Au décours du travail psychothérapeutique, Malvin pourra se risquer à des évocations qui témoignent, en négatif, de la proximité de ses préoccupations génitales, dont on peut faire l'hypothèse qu'elles se trouvent soutenues par une affirmation de ses assises narcissiques-identitaires.

En effet, Malvin formule à différentes reprises que les relations avec les jeunes filles ne l'intéressent pas: il n'a pas de temps pour cela, et en tous les 
cas il doit selon lui attendre de grandir (il faut entendre «vieillir», quitter l'enfance et le registre d'investissement de ses objets qui la caractérise) pour être en mesure d'investir une relation amoureuse. Lors d'une séance, après un peu plus de deux années de psychothérapie, Malvin indique que ses amis se préoccupent de sa vie affective. L'un d'eux lui a présenté sa cousine, âgée de 3 ans de plus que Malvin ( «une vieille» dira l'adolescent). Cet ami fait en sorte que Malvin rencontre régulièrement cette jeune femme, mais Malvin résiste, il dit qu'il verra plus tard, et que pour le moment il doit suivre sa formation et réussir ses diplômes. Cette situation semble placer Malvin dans une forme de redoublement d'un vécu de passivation: tout comme la puberté confronte l'adolescent à la passivité, la sollicitation amoureuse (et sexuelle), à l'initiative d'un pair, le contraint et fait traumatisme. Mais pour l'heure, l'investissement intellectuel demeure pour Malvin une voie dérivative de la pulsion sexuelle, sur des modalités proches de ce que l'on peut observer dans le temps de la latence.

Comment penser cette offre sexuelle, dans un registre génital, adressée à Malvin? Cette offre provient d'un pair, un ami de son âge, support des identifications homomorphes, qui lui propose une relation avec sa cousine, dans une forme de marché sexuel. On imagine les fantasmes qui sous-tendent cette offre: figure de la passivité féminine, investie comme un objet dont le marché pourrait être géré par les garçons; figure de l'incestuel, portée par la préoccupation et la contribution active de l'ami de Malvin pour la vie amoureuse de sa cousine, figure de l'offre homosexuelle sous-tendue par cette manœuvre de séduction... Par ailleurs, on peut interroger la manière dont cette offre s'inscrit dans le statut des investissements œedipiens de Malvin. On a pu en effet reconnaître la difficulté de Malvin à se détacher du premier objet d'investissement que représente la figure maternelle: elle est en effet tout à la fois prise dans un lien d'emprise idéalisé (le versant de la figure héroïque de la mère) et dans la rencontre de sa défaillance essentielle (le versant de la figure de l'abandon, vécu par Malvin dans sa petite enfance et, plus tard, par identification, à l'égard du jeune enfant de la fratrie). Mais ces deux figures restent clivées, dans une construction ambivalente fragile, comme cela a été relevé. Comment dans ce contexte envisager un détachement suffisant de l'objet primaire pour investir un nouvel objet, potentiellement rival? Comment soutenir la réactivation des fantasmes incestueux appelés par un investissement amoureux? Comment prendre le risque d'un nouvel abandon de la part de sa mère, qui pourrait intervenir comme punition d'un défaut de loyauté de Malvin? Enfin, sur quelle figure 
d'identification masculine construire l'investissement amoureux? Dans ce contexte, on peut penser qu'il est plus économique pour Malvin de se tenir à distance de sa pulsionnalité et de son engagement dans une sexualité génitale, dangereuse à bien des égards.

\section{Le clivage et ses destins...}

Témoin d'une identité en souffrance, le narcissisme blessé se déploie sur fond de clivage. Ce clivage prend la forme subtile du clivage au moi (Roussillon, 1999), qui assure paradoxalement une coexistence au sein de l'appareil psychique entre des contenus symbolisés et non symbolisés; il se déploie secondairement en direction d'un clivage des objets, ici, dans la tension entre les valences de l'héroïsme et de l'impuissance des figures parentales. Le clivage au moi atteint la qualité des liaisons représentatives (de l'affect à la représentation) et maintient à distance, dans un statut nonsymbolisé, les éprouvés traumatiques de l'infans, réactivés dans le temps du pubertaire. On peut en identifier tout particulièrement la trace au travers des effets d'oubli dont témoigne de manière récurrente Malvin, effets d'oubli qui, s'il se montre indifférent à leur égard au début du traitement, vont progressivement lui être inconfortables, signe d'une levée (progressive et partielle) du clivage.

Dans ce contexte, on peut considérer que le narcissisme blessé contient la trace de ces éprouvés non-symbolisés des expériences précoces (abandon, délaissement, empiétements, harcèlement) qui portent atteinte à la consistance identitaire. Stratégie de protection au service du moi, le clivage contribue à ordonner les différentes valences du fonctionnement psychique de Malvin et des contenus qui l'habitent. À ce titre, il constitue un indicateur précieux pour la conduite du traitement psychothérapeutique comme contribution à la restauration des parcelles trouées de sa subjectivité. Malvin peut progressivement faire part de l'ambivalence de ses mouvements internes sans redouter la rétorsion ou l'effondrement, et prendre appui sur un cadre suffisamment bienveillant pour accueillir ses incertitudes, $y$ compris pour ce qui concerne la qualité de ses investissements dans le soin. Au plan contre-transférentiel, l'assouplissement des positions clivées peut se lire dans la restauration d'un sentiment davantage apaisé dans la rencontre avec l'adolescent: la possible liaison des éprouvés d'incompréhension à l'égard de pans entiers de sa subjectivité (dont on peut penser qu'ils venaient utilement contenir et/ou masquer les éprouvés de haine sous-tendus par les multiples replis subjectifs de Malvin) à des éprouvés davantage bienveillants (à même d'accueillir la 
destructivité contenue dans les agirs violents sexuels et dans le déni subjectif qui les accompagne) peut être considérée comme le témoin d'une évolution processuelle qui signe l'amorce d'une relance de la subjectivation.

Le travail du clivage est à ce prix, qui autorise la réinstauration d'une suffisante continuité identitaire au décours du jeu ouvert par le transfert, au service de la subjectivité. Comme le soutient Freud (1914), le transfert autorise une voie de dégagement au regard des effets de répétition:

Or le moyen principal de dompter la contrainte de répétition du patient et de la transformer en un motif de remémoration se trouve dans le maniement du transfert [...]. Nous lui ouvrons avec le transfert un lieu d'ébats où il lui est permis de se déployer dans une liberté presque totale et où il lui est assigné de nous mettre sous les yeux tout ce qui, en fait de pulsions pathogènes, s'est caché dans la vie d'âme de l'analysé. (Freud, 1914, p. 194).

La clinique de Malvin invite à penser les enjeux de répétition traumatique qui se révèlent dans la scène sexuelle transgressive et qui, à leur corps défendant, s'infiltrent dans le jeu transférentiel. Tout se passe comme si la scène des violences sexuelles ouverte par Malvin venait tout à la fois dire et obturer le secret d'une impasse dans le lien mère-enfant, sous la double forme de la défaillance et de l'excitation, dont le travail psychothérapeutique autorise le dégagement, au service d'un processus de soutien et/ou de restauration identitaire. L'appui sur le psychothérapeute, y compris sur les défaillances de celui-ci à maintenir de manière suffisamment constante un investissement créatif face aux expressions mortifères des mouvements psychiques, autorise pour Malvin une forme de relance dans le travail de subjectivation. On ne peut méconnaître, enfin, la charge des enjeux liés à la différence culturelle, qui, avec Kaës (1998), peut être pensée dans l'articulation entre identité en souffrance à l'adolescence et «souffrances de l'identité»... mais cela ouvrirait encore à un autre chapitre de l'histoire.

\author{
Pascal Roman \\ pascal.roman@unil.ch
}

\title{
Notes
}

1. Gutton (1991) a recours à deux formulations, le «gel du pubertaire» et le "gel du processus pubertaire», auxquelles je fais ici référence: «résistance au pubertaire susceptible d'aller jusqu'à sa neutralisation» (p. 213), qui implique un «sacrifice de l'éprouvé pubertaire» (p. 214). Ainsi, le gel du (processus) pubertaire renvoie à des situations 
cliniques comme celle de Malvin, dans lesquelles la «scène pubertaire ne peut se jouer» (p. 211).

2. Tout comme on peut lire, au bord des voies ferrées ou à l'aplomb des passages à niveau: "Un train peut en cacher un autre.»

3. "L'agir violent grave vient "faire évènement", un évènement irréversible en lieu et place d'un temps irréversible qu'engagerait la puberté. Somme toute, un évènement qui peut potentiellement générer une forme de temporalité» (Marro, 2019, p. 25).

\section{Références}

Cahn, R. (2004). Subjectalité et subjectivation. Adolescence, 50 (4), 755-766.

Ciavaldini, A. (2006). La pédophilie, figure de la dépression primaire. Revue française de psychanalyse, 70 (1), 177-195.

Freud, S. (1905). Trois essais sur la théorie sexuelle. Dans Euvres complètes VI (p. 59-181). Paris: Presses universitaires de France.

Freud, S. (1914). Remémoration, répétition et élaboration. Dans Euvres complètes XII (p. 185-196). Paris: Presses universitaires de France.

Freud, S. (1926). Inhibition, symptôme et angoisse. Dans Euvres complètes XVII (p. 203 286). Paris: Presses universitaires de France.

Gutton, P. (1991). Le pubertaire. Paris: Presses universitaires de France.

Jeammet, P. (1994). Adolescence et processus de changement. Dans D. Widlöcher (dir.), Traité de psychopathologie (p. 687-726). Paris: Presses universitaires de France.

Kaës, R. (1998). Différence culturelle et souffrances de l'identité. Paris: Dunod.

Kaës, R. (2012). Le malêtre. Paris: Dunod.

Lacan, J. (1949). Le stade du miroir comme formateur de la fonction du Je. Dans Écrits I (p. 89-97). Paris: Seuil, 1966.

Laplanche, J. et Pontalis, J.-B. (1967). Vocabulaire de la psychanalyse. Paris: Presses universitaires de France.

Marro, J. (2019). Agirs hétéro-agressifs graves à l'adolescence et temporalité psychique. Psychothérapies, 39 (1), 21-28.

Roman, P. (2018). L'inadvenu de l'affect et la trace du traumatisme dans les violences sexuelles à l'adolescence. Adolescence, 36 (1), 109-120.

Roman, P. et Ravit, M. (2006). La violence sexuelle dans la famille et la mise à l'épreuve des liens fraternels. Cahiers de psychologie clinique, 27, 11-27.

Roussillon, R. (1999). Agonie, clivage et symbolisation. Paris: Presses universitaires de France. Winnicott, D. W. (1956). La préoccupation maternelle primaire. Dans De la pédiatrie à la psychanalyse (p. 168-174). Paris: Payot, 1975.

Winnicott, D. W. (1956). La tendance antisociale. Dans Déprivation et délinquance (p. 145158). Paris: Payot. 